\title{
Temperature-difference-driven mass transfer through the vapor from a cold to a warm liquid
}

\author{
Henning Struchtrup, ${ }^{1, *}$ Signe Kjelstrup, ${ }^{2,3}$ and Dick Bedeaux ${ }^{2}$ \\ ${ }^{1}$ Department of Mechanical Engineering, University of Victoria, Victoria, Canada, BC V8W 3P6 \\ ${ }^{2}$ Department of Chemistry, Norwegian University of Science and Technology, 7491 Trondheim, Norway \\ ${ }^{3}$ Process and Energy Laboratory, Delft University of Technology, Leeghwaterstraat 44, 2628 CA Delft, The Netherlands \\ (Received 9 January 2012; revised manuscript received 22 February 2012; published 4 June 2012)
}

\begin{abstract}
Irreversible thermodynamics provides interface conditions that yield temperature and chemical potential jumps at phase boundaries. The interfacial jumps allow unexpected transport phenomena, such as the inverted temperature profile [Pao, Phys. Fluids 14, 306 (1971)] and mass transfer from a cold to a warm liquid driven by a temperature difference across the vapor phase [Mills and Phillips, Chem. Phys. Lett. 372, 615 (2002)]. Careful evaluation of the thermodynamic laws has shown [Bedeaux et al., Physica A 169, 263 (1990)] that the inverted temperature profile is observed for processes with a high heat of vaporization. In this paper, we show that cold to warm mass transfer through the vapor from a cold to a warm liquid is only possible when the heat of evaporation is sufficiently small. A necessary criterium for the size of the mass transfer coefficient is given.
\end{abstract}

DOI: 10.1103/PhysRevE.85.061201

PACS number(s): 05.70.Np, 05.70.Ln, 64.70.fm

\section{INTRODUCTION}

Conditions for conversion of liquid to vapor, and vice versa, are of vital importance in a majority of industrial and natural processes. In dynamic studies of phase transitions, it has become increasingly clear that the interface and the interface region can play a decisive role in determining the rate of transformation. This paper concerns the importance of jumps in temperature and chemical potential at a vapor-liquid interface and, in particular, the role of these jumps in the determination of the mass and heat flows [1].

A recent experimental paper has suggested the possibility for transfer of mass from a cold to a warm liquid reservoir via a vapor phase [2]. Specifically, the experiments are conducted in systems where the temperature difference across the system is the only driving force, see Fig. 1 (left panel) for an illustration of the setting and the direction of fluxes in this case.

It is not obvious that mass transfer against a temperature difference is in agreement with the second law of thermodynamics, and it is one aim of this paper to clarify the conditions for such transport in terms of a criterion for system properties.

The problem has some similarity to the problem of the inverted temperature profile discussed earlier [3-5]. In that problem, solved by kinetic theory $[3,4]$ as well as nonequilibrium thermodynamics [5,6], a temperature gradient is formed in a vapor layer, which is sandwiched between two liquid (or solid) layers in a direction opposite the overall temperature gradient, cf. Fig. 1 (right panel) for flux directions.

We will see that the observation of cold to warm mass transfer as well as the inverted temperature profile will depend on the size of the heat of evaporation. We show here that cold to warm mass transfer may be observed for low heats of evaporation. It is already known that the inverted temperature profile requires a high heat of vaporization [6]. We will generalize the form of the last criterion somewhat.

\footnotetext{
*struchtr@uvic.ca
}

\section{SYSTEM CONFIGURATION}

The system of interest is, in both cases, contained in a closed chamber of large base area and low height so that the transport processes are essentially one dimensional and in the vertical direction. The upper and lower plates that enclose the chamber are thermostatted by circulating fluids above and below. The chamber is filled with a substance, cleaned and degassed so that only the pure substance is contained in the chamber. Filling amount and temperatures are such that some of the substance is liquid and the remainder is in the vapor phase. The temperature $T_{L}$ of the bottom plate is kept constant, and the temperature $T_{H}$ of the upper plate is varied where $T_{L}<T_{H}$. The question is whether liquid can be transferred from the cold lower to the warm upper plate when the temperature difference is the only driving force.

Criteria can be obtained by solving transport equations for heat and mass in the three bulk layers and the corresponding two boundary layers involved. This was performed to determine that the existence of an inverted temperature profile did not violate the second law of thermodynamics $[5,6]$ and was also performed here. We use standard linear nonequilibrium thermodynamics to obtain the criteria. It is sufficient to consider a system close to global equilibrium. The completely linearized form of the equations can then be used to find a solution, and this simplifies derivations. The result in each case is given in terms of an inequality, which is amenable to an experimental test. It will be compared with data from the literature.

Figure 1 shows a schematic of the cell with two layers of liquid (thicknesses $x_{L}$ and $\Delta$ ) separated by a vapor layer (thickness $x_{V}$ ). The liquids close to the bottom and to the upper plates assume the temperatures of the plates. We have highlighted these temperatures $T_{L}$ and $T_{H}$. The temperatures $T_{l}$ and $T_{v}$ are those of the liquid and vapor at the lower phase boundary, and the temperatures $T_{V}$ and $T_{\Delta}$ are those of the vapor and liquid at the upper phase boundary. We consider a case where the upper plate is fully wetted. When $T_{H}$ is kept equal to $T_{L}$, the system is in equilibrium.

The cited experiments [2] are conducted in fully closed cells where the process is transient since liquid is removed 

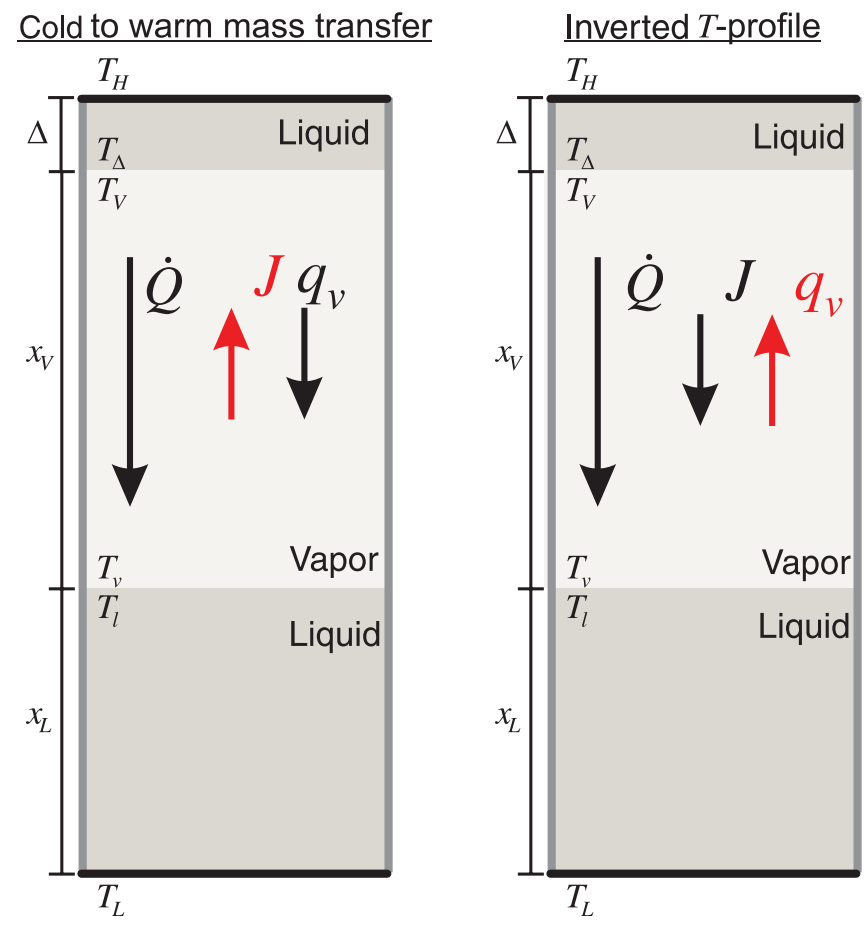

FIG. 1. (Color online) Directions of mass and energy fluxes in left: cold to warm mass transfer and in right: the inverted temperature profile. Two layers of liquid (thickness $x_{L}$ and $\Delta$ ) are separated by a vapor layer (thickness $x_{V}$ ). Temperatures $T_{L}$ and $T_{H}$ apply to chamber walls, temperatures $T_{l}$ and $T_{v}$ apply to liquid and vapor at the lower phase boundary, and temperatures $T_{V}$ and $T_{\Delta}$ apply to vapor and liquid at the upper phase boundary.

from one layer and is deposited onto the other so that the layer thicknesses change over time. In order to simplify the computations, we evaluate an equivalent steady state process where the liquid that is removed is replenished at the plate with the temperature of the plate and liquid that is deposited is removed at the corresponding plate. The important point in the experiment is that the transport is driven only by the temperature difference between the plates, and this is the case for our steady state process. Since the observed mass transfer rate and, thus, the change in layer thickness is extremely small, the two systems and, in particular, the interfaces agree at a point in time when the layer thicknesses are the same.

In recent years, Fang and Ward [7-9], Badam et al. [10], and Duan et al. [11] published a number of papers on experiment and theory of nonequilibrium interfaces. Their experiments differ fundamentally from the setup described here: In our setup, the vapor is sandwiched between two liquid surfaces. The only driving force for the transfer of mass and energy is the temperature difference imposed between the upper and the lower boundaries. Moreover, the pressure in the cell is not controlled. In their experiments, on the other hand, the vapor sits on top of a single liquid layer. For most experiments, the outer system boundary is kept at constant temperature, but there are also some experiments with additional heating. The main driving force is the deviation in pressure from the saturation pressure, which is controlled by means of a vacuum pump. For experiments with heating [10,11], there are two forces, the pressure deviation and the temperature gradient. Mass transfer from cold liquid to warm vapor is forced by the vacuum pump. None of these experiments address the possibility of mass transfer from a cold to a warm liquid through the vapor driven only by the temperature difference. A thorough evaluation of these experiments based on nonequilibrium thermodynamics was presented in Ref. [12].

\section{THERMODYNAMIC DESCRIPTION}

We consider only small changes in the stationary state in $T_{H}$ away from $T_{L}$ and fully linearize the whole description around their differences. In equilibrium, all fluxes are zero. Away from equilibrium, the fluxes are, therefore, of linear order in $T_{H}-T_{L}$. For linear order, all transport coefficients have their equilibrium values. The position of the liquid-vapor interfaces are chosen to be the equimolar or, equivalently, equimass dividing surfaces [1].

In the stationary state, the mass flux $J$ is constant throughout the three layers. As the velocities are linear in the temperature difference, for linear order, one may obtain them by dividing $J$ by the corresponding equilibrium mass density for each of the three bulk layers. It follows that the velocities are constant in all three layers and that they are equal in the liquid layers. When velocities are constant, there is no viscous contribution to the pressure. It follows that the hydrostatic pressure $p$ is constant throughout the system. Under stationary state conditions, the liquid layer thicknesses $x_{L}$ and $\Delta$ are also constant. Evaporation on one side of the system and condensation on the other side means, therefore, continuous replenishing of liquid at the side where evaporation occurs and removal of liquid at the side where condensation occurs. When the temperatures $T_{L}$ and $T_{H}$ differ only slightly, the flux $J$ through the cell is small so that an experiment with replenishment and removal of liquid is very close to an experiment in a closed system where no mass enters or leaves the cell but where the thicknesses of the liquid's layers change.

The thermal energy is conserved, making the energy flux $\dot{Q}$ a constant through the three layers where $\dot{Q}=h J+$ $q, h$ being the specific enthalpy density and $q$ being the measurable heat flux. In the approximation for linear order in the overall temperature difference, we should use the equilibrium enthalpies in the liquid layers $h_{\ell}\left(T_{L}\right)=h_{0}$, and in the vapor $h_{v}\left(T_{L}\right)=h_{f g}^{L}+h_{0}$, where $h_{f g}^{L}$ is the specific heat of evaporation. For convenience, we choose a reference enthalpy such that $h_{0}=0$, making $\dot{Q}=q_{\ell}$. The measurable heat fluxes in the liquid $q_{\ell}=-\kappa_{L} d T / d x$ and in the vapor $q_{v}=-\kappa_{V} d T / d x$ are also constant at linear order. Here, $\kappa_{L}$ and $\kappa_{V}$ are the equilibrium thermal conductivities. As a consequence, the temperature gradients in the three layers are also constant at linear order.

For the given geometry, the second law of thermodynamics states that the divergence of the entropy flux must be nonnegative,

$$
\frac{d}{d x}\left[J s(T)+\frac{q}{T}\right] \geqslant 0 .
$$

As we explained above, $J$ is constant everywhere, whereas, $q$ is constant in the three layers. For linear order, one should furthermore use the equilibrium specific entropy densities in 
the liquid and the vapor. Upon integration over the three layers, using standard thermodynamic relations, this gives, for the total entropy production,

$$
q_{\ell}\left(\frac{1}{T_{H}}-\frac{1}{T_{L}}\right) \geqslant 0
$$

valid up to second order in the temperature difference $\left(T_{H}-\right.$ $T_{L}$ ). Equation (2) contains the measurable heat flux in the liquid $q_{\ell}=\dot{Q}-J h_{0}=\dot{Q}$ and does, as required, not depend on the choice of the reference value for the enthalpy. The integrated energy balance in the three bulk layers becomes, for the bottom liquid $\left(0, x_{L}\right)$,

$$
\dot{Q}=q_{\ell}=-\kappa_{L} \frac{T_{l}-T_{L}}{x_{L}}
$$

for the vapor $\left(x_{L}, x_{V}\right)$,

$$
\dot{Q}=J h_{f g}^{L}+q_{v}=J h_{f g}^{L}-\kappa_{V} \frac{T_{V}-T_{v}}{x_{V}},
$$

and for the upper liquid $\left(x_{L}+x_{V}, x_{L}+x_{V}+\Delta\right)$,

$$
\dot{Q}=q_{\ell}=-\kappa_{L} \frac{T_{H}-T_{\Delta}}{\Delta} .
$$

We refer to $\dot{Q}$, which is constant throughout the system, and which determines the total entropy production, as the overall energy flow.

To determine the full solution, we need the conditions provided by classical irreversible thermodynamics $[1,5,13]$ for both interfaces. For small differences in temperatures, we have, for the lower interface,

$$
\begin{aligned}
\frac{p_{\mathrm{sat}}\left(T_{l}\right)-p}{\sqrt{2 \pi R T_{L}}} & =\hat{r}_{11} J+\hat{r}_{12} \frac{q_{v}}{R T_{L}}, \\
\frac{p_{\mathrm{sat}}\left(T_{L}\right)}{\sqrt{2 \pi R T_{L}}} \frac{T_{l}-T_{v}}{T_{L}} & =\hat{r}_{21} J+\hat{r}_{22} \frac{q_{v}}{R T_{L}},
\end{aligned}
$$

where $p_{\text {sat }}(T)$ is the saturation pressure at temperature $T$ and $R=R_{u} / M$ is the gas constant per unit of mass $\left(R_{u}\right.$ is the universal gas constant, and $M$ is the molecular weight). The above equations are written such that the Onsager resistivities $\hat{r}_{\alpha \beta}$ are dimensionless [13]. The second law of thermodynamics requires the resistivity matrix to be non-negative definite, $\hat{r}_{11} \geqslant$ $0, \hat{r}_{22} \geqslant 0, \hat{r}_{11} \hat{r}_{22}-\hat{r}_{12} \hat{r}_{21} \geqslant 0[1,14]$. Due to the microscopic reversibility of the evaporation and condensation processes, the Onsager reciprocity relations hold [15], which state that the resistivity matrix is symmetric. While molecular dynamics experiments hint at symmetry [16], the scatter in the data does not allow a firm statement, see the discussion in Ref. [13]. Nevertheless, the arguments for Onsager symmetry are strong and convincing, and thus, we assume it from now on, that is, we set $\hat{r}_{21}=\hat{r}_{12}$.

The heat of transfer of the surface is defined by

$$
\frac{q_{f g}^{*}}{R T_{L}} \equiv \frac{1}{R T_{L}}\left(\frac{q_{v}}{J}\right)_{T_{v}=T_{l}}=-\frac{\hat{r}_{12}}{\hat{r}_{22}} .
$$

The values of the resistivities depend on the material and the temperature. If the vapor behaves as an ideal gas with constant condensation coefficient $\psi$, it follows, using kinetic theory $[1,17]$, that the dimensionless resistivities $\hat{r}_{\alpha \beta}$ are constants with the values,

$$
\hat{r}_{\alpha \beta}=\left[\begin{array}{cc}
\frac{1}{\psi}-0.40044 & 0.126 \\
0.126 & 0.294
\end{array}\right] .
$$

The resulting heat of transfer $q_{f g}^{*}=-0.429 R T_{L}$ is negative when the vapor behaves as an ideal gas.

According to the interface conditions (6), mass and/or heat transfer through an interface lead to temperature differences between liquid and vapor and deviations from the saturation pressure. Molecular dynamics simulations for simple twophase fluids of particles with a short attractive potential confirm the values found using kinetic theory [18]. For longer-range potentials, the diagonal coefficients decrease a bit, whereas, the coupling coefficient increases $[1,19]$. The resulting heat of transfer becomes about a factor of 3 larger compared to the value from kinetic theory and is still negative.

In special geometries, temperature jumps of up to $8^{\circ}$ were found in open system experiments where the pressure was controlled by pumping vapor out $[7-9,11,12]$. Such jumps require values of the interfacial resistances between 1 and 2 orders larger than those found using kinetic theory [20]. In other experiments $[10,11]$, temperature jumps of more than $20^{\circ}$ were found. In particular, in smaller systems, jumps in the temperature and deviations from the saturation pressure can be significant compared to other temperature and pressure differences.

The corresponding conditions for the upper interface read

$$
\begin{aligned}
\frac{p-p_{\mathrm{sat}}\left(T_{\Delta}\right)}{\sqrt{2 \pi R T_{L}}} & =\hat{r}_{11} J+\hat{r}_{12} \frac{q_{v}}{R T_{L}}, \\
\frac{p_{\text {sat }}\left(T_{L}\right)}{\sqrt{2 \pi R T_{L}}} \frac{T_{V}-T_{\Delta}}{T_{L}} & =\hat{r}_{12} J+\hat{r}_{22} \frac{q_{v}}{R T_{L}} .
\end{aligned}
$$

The signs of fluxes relative to the interface are inverted compared to the lower interface. The heats of transfer of the upper and the lower interfaces are the same in the fully linearized case, and the saturation pressures at $T_{l}$ and $T_{\Delta}$ must be expanded around $T_{L}$ as

$$
p_{\text {sat }}(T)=p_{\text {sat }}\left(T_{L}\right)\left[1+\hat{Z} \frac{h_{f g}^{L}}{R T_{L}} \frac{T-T_{L}}{T_{L}}\right] .
$$

The Clausius-Clapeyron relation was used to express the derivative of the saturation pressure as

$$
\frac{d \ln p_{\mathrm{sat}}\left(T_{L}\right)}{d \ln T}=\hat{Z} \frac{h_{f g}^{L}}{R T_{L}}, \quad \hat{Z}=\frac{R T_{L}}{p_{\mathrm{sat}}\left(T_{L}\right)\left(v_{g, L}-v_{f, L}\right)},
$$

with $v_{g, L}$ and $v_{f, L}$ as the specific volumes of saturated vapor and liquid at $T_{L}$. The coefficient $\hat{Z}$ is dimensionless. Far from the critical point (cr), the liquid volume can be ignored against the vapor volume, and the vapor behaves as an ideal gas so that $\hat{Z}=1$. Approaching the critical point, the value of $\hat{Z}$ increases toward infinity, and the latent heat vanishes. The slope of the saturation pressure stays finite so that $\left[\hat{Z} h_{f g}^{L}\right]_{\mathrm{cr}}$ is finite.

\section{SOLUTION}

Equations (3)-(6) and (9) provide a linear system of seven equations for the seven unknowns $T_{l}, T_{v}, T_{V}, T_{\Delta}, \dot{Q}, J$, and $p$. The solution for $J$ and $\dot{Q}$ is cumbersome but straightforward. The resulting lengthy expressions are best written with 
several abbreviations,

$$
\begin{gathered}
J=\frac{-A}{2[C+D]+E B}\left[\frac{p_{\mathrm{sat}}\left(T_{L}\right)}{T_{L} \sqrt{2 \pi R T_{L}}}\left(T_{H}-T_{L}\right)\right], \\
\dot{Q}=\frac{-B}{2[C+D]+E B}\left[\frac{p_{\mathrm{sat}}\left(T_{L}\right) R}{\sqrt{2 \pi R T_{L}}}\left(T_{H}-T_{L}\right)\right],
\end{gathered}
$$

where

$$
\begin{aligned}
& A=\hat{Z} \frac{h_{f g}^{L}}{R T_{L}}\left(\frac{1}{2} \frac{x_{V}}{\lambda_{0}}+\hat{r}_{22}\right)-\hat{r}_{12}, \\
& B=\hat{Z} \frac{h_{f g}^{L}}{R T_{L}}\left[\frac{h_{f g}^{L}}{R T_{L}}\left(\frac{1}{2} \frac{x_{V}}{\lambda_{0}}+\hat{r}_{22}\right)-\frac{\hat{Z}+1}{\hat{Z}} \hat{r}_{12}\right]+\hat{r}_{11}, \\
& C=\hat{r}_{11} \frac{1}{2} \frac{x_{V}}{\lambda_{0}} \geqslant 0, \quad D=\hat{r}_{11} \hat{r}_{22}-\hat{r}_{12}^{2} \geqslant 0, \\
& E=\frac{\kappa_{V}}{\kappa_{L}} \frac{x_{L}+\Delta}{\lambda_{0}} \geqslant 0 .
\end{aligned}
$$

From their definitions, it is clear that $C, D$, and $E$ are always positive. The signs for $A$ and $B$ will, therefore, lead to the criterion for cold to warm mass transfer.

In the above result, the characteristic length scale for heat transfer in the vapor appears, which is defined as

$$
\lambda_{0}=\frac{\kappa_{V} \sqrt{2 \pi R T_{L}}}{p_{\mathrm{sat}}\left(T_{L}\right) R} .
$$

This length scale is on the order of several mean free paths of the vapor. For a vapor layer of a macroscopic thickness so that $x_{V} \gg \lambda_{0}$, its total resistance is much larger than the interfacial resistivities. The resistivity for heat transfer in the vapor then plays the dominant role for the values of $J$ and $\dot{Q}$. In this case, $A$ and $B$ are positive so that the energy flux $\dot{Q}$ and mass flux $J$ both go from warm to cold, i.e., the usual warm to cold mass transfer is observed. Only if the thickness of the vapor layer is on the order of $2 \lambda_{0} \hat{r}_{22}$, do the interfacial resistivities have a comparable influence on the mass and energy transport across the cell. It is important to realize that the condition $x_{V} \gg 2 \lambda_{0} \hat{r}_{22}$ does not imply that the interfacial resistances can be ignored in the calculation of the temperature and pressure jumps at the liquid-vapor surfaces. Both the size and the sign of $q_{v}$ depend crucially on the interface conditions [21]. The inverted temperature profile, for which $q_{v}>0$, occurs also when $x_{V}$ is large.

\section{ENERGY FLOW}

We first consider the direction of the overall energy flow $\dot{Q}-J h_{0}=\dot{Q}$, which, according to the second law (2), must point from warm to cold. In the present geometry, this means that the dimensionless factor in (13) must be positive. Since the coefficients $C, D$, and $E$ are always positive, the second law requires $B \geqslant 0$. By completing the square and some manipulation, this condition can be written as

$$
\begin{aligned}
& \left(\frac{h_{f g}^{L}}{R T_{L}}\right)^{2} \frac{1}{2} \frac{x_{V}}{\lambda_{0}}+\frac{1}{\hat{Z}} \frac{\hat{r}_{11} \hat{r}_{22}-\hat{r}_{12}^{2}}{\hat{r}_{22}} \\
& \quad+\hat{r}_{22}\left\{\left[\frac{\hat{Z}+1}{2 \hat{Z}} \frac{\hat{r}_{12}}{\hat{r}_{22}}-\frac{h_{f g}^{L}}{R T_{L}}\right]^{2}-\left[\frac{\hat{Z}-1}{2 \hat{Z}} \frac{\hat{r}_{12}}{\hat{r}_{22}}\right]^{2}\right\}>0 .
\end{aligned}
$$

The first two terms are positive $\left(D \geqslant 0, \hat{r}_{22} \geqslant 0\right)$. Far from the critical point where $\hat{Z} \simeq 1$, the expression in the curly brackets is positive so that the above inequality is always fulfilled. Close to the critical point $h_{f g}^{L} \rightarrow 0$ and $\hat{Z} \rightarrow \infty$, but their product remains finite, this implies that the expression in curly brackets goes to zero. Furthermore, as the interfacial resistances go to zero, only the first term remains, which is positive. Thus, $B \geqslant 0$, that is, the overall energy flux is always from the hot to the cold liquid.

\section{MASS FLOW}

Consider now the possibility for warm to cold mass transfer far from the critical point. Evaporation and subsequent condensation at a warmer liquid means that the mass flux is directed opposite the heat flux. Irreversible thermodynamics does not exclude this phenomenon, for which $J>0$. Equation (12) shows that this is the case for $A<0$ or

$$
\hat{Z} \frac{h_{f g}^{L}}{R T_{L}}\left[\frac{1}{2} \frac{x_{V}}{\lambda_{0}}+\hat{r}_{22}\right]<\hat{r}_{12} .
$$

Using the heat of transfer of the liquid-vapor interface, Eq. (7), this inequality can be written as

$$
\hat{Z} h_{f g}^{L}<\left(-q_{f g}^{*}\right) \frac{\hat{r}_{22}}{\frac{1}{2} \frac{x_{V}}{\lambda_{0}}+\hat{r}_{22}} .
$$

When the vapor is close to ideal $(\hat{Z}=1)$, kinetic theory gives $\left(-q_{f g}^{*}\right)=0.429 R T_{L}$. The factor $\left(x_{V} / 2 \lambda_{0}\right)+\hat{r}_{22}$ is the integrated dimensionless resistance of half the vapor layer plus one liquid-vapor interface. For an ideal vapor next to its liquid, one always has $h_{f g}^{L}>\left(-q_{f g}^{*}\right)$. The last factor is clearly smaller than unity. It follows that cold to warm mass transfer is not possible far from the critical point, independent of the thickness of the ideal vapor layer when interface resistivities are given by kinetic theory [18].

For more realistic systems [19], the diagonal resistivities decreased a bit, whereas, the heat of transfer increased by a factor near 3. This increase is not enough to make cold to hot mass transfer possible. The few experiments that, so far, are available [9], seem to indicate [20] that the diagonal resistivities are between 1 and 2 orders of magnitude larger than those predicted by kinetic theory. This surprising difference still needs to be clarified. For this reason, we are not able to fully reject the possibility of cold to warm mass transfer far away from the critical point.

Nevertheless, the criterion (17) leads to some further insight, related to the layer thickness: Cold to warm mass transfer might be possible in vapor layers where the thickness $x_{V}$ obeys

$$
x_{V}<2 \lambda_{0} \hat{r}_{22}\left[\frac{\left(-q_{f g}^{*}\right)}{\hat{Z} h_{f g}^{L}}-1\right] .
$$

According to (19), the thickness $x_{V}$ is on the order of the length scale times the coupling coefficient for heat transfer. The value for water is $\lambda_{0}=0.053 \mathrm{~mm}$ at $T=273 \mathrm{~K}$ and $\lambda_{0}=$ $7 \times 10^{-6} \mathrm{~mm}$ at the critical point. The product $\lambda_{0} \hat{r}_{22}$ must be large enough to have an observable vapor layer. Furthermore, the expression on the right hand side of (19) must be positive to allow for cold to warm mass transfer. A necessary criterion 
is, therefore,

$$
\frac{d \ln p_{\mathrm{sat}}\left(T_{L}\right)}{d \ln T_{L}}=\hat{Z} \frac{h_{f g}^{L}}{R T_{L}}<\frac{\left(-q_{f g}^{*}\right)}{R T_{L}} .
$$

For water, the expression $\hat{Z} h_{f g}^{L} / R T_{L}$ assumes values from 20 at the triple point $T_{\text {tr }}=273 \mathrm{~K}$ to 7 at the critical point $T_{\text {cr }}=647 \mathrm{~K}$. With the reported values of $\hat{Z} h_{f g}^{L} / R T_{L}$, the dimensionless absolute heat of transfer $\left(-q_{f g}^{*}\right) / R T_{L}$ would have to be larger than these values to make $x_{V}$ positive. Close to the critical point, $\hat{Z} h_{f g}^{L} / R T_{L}$ is smaller, and the resistivities vanish; the heat of transfer goes probably to a constant value.

We are not aware of systematic measurements that were aimed directly at determining the interface coefficients, and thus, we have no reliable data for evaluating the inequality (17) in detail. Nevertheless, we can make some basic considerations that indicate that it is quite unlikely (if not impossible) that cold to warm mass transfer is observable in experiments. For this, we assume that, indeed, the inequality (17) is fulfilled by setting $\hat{r}_{12}>\hat{Z} \frac{h_{f g}^{L}}{R T_{L}}\left(\frac{1}{2} \frac{x_{V}}{\lambda_{0}}+\hat{r}_{22}\right)$. The second law for the interface requires $D=\hat{r}_{11} \hat{r}_{22}-\hat{r}_{12} \hat{r}_{12} \geqslant 0$. Combining both requirements results in an inequality relation between $\hat{r}_{22}$ and $\hat{r}_{11}$, viz.,

$$
\hat{r}_{11} \geqslant\left(\hat{Z} \frac{h_{f g}^{L}}{R T_{L}}\right)^{2}\left[\frac{1}{4 \hat{r}_{22}}\left(\frac{x_{V}}{\lambda_{0}}\right)^{2}+\frac{x_{V}}{\lambda_{0}}+\hat{r}_{22}\right] .
$$

For given temperature and cell dimensions, the right hand side of the inequality is an algebraic function in $\hat{r}_{22}$, with a minimum at $\hat{r}_{22 \mid \min }=\frac{1}{2} \frac{x_{V}}{\lambda_{0}}$. It follows that the smallest possible value for $\hat{r}_{11}$ that allows for cold to warm mass transfer is the minimum of the function on the right,

$$
\hat{r}_{11} \geqslant 2\left(\hat{Z} \frac{h_{f g}^{L}}{R T_{L}}\right)^{2} \frac{x_{V}}{\lambda_{0}} .
$$

We evaluate this condition with data for water at $T_{L}=$ $2.5^{\circ} \mathrm{C}$, where $p_{\text {sat }}\left(T_{L}\right)=730 \mathrm{~Pa}, \hat{Z} \simeq 1, \frac{h_{f g}^{L}}{R T_{L}}=19.6$, and $\kappa_{V}=0.0165 \mathrm{~W} / \mathrm{mK}$ so that $\lambda_{0}=4.376 \times 10^{-5} \mathrm{~m}$. We find

$$
\hat{r}_{11}>2\left(\hat{Z} \frac{h_{f g}^{L}}{R T_{L}}\right)^{2}\left(\frac{x_{V}}{\lambda_{0}}\right) \simeq \frac{x_{V}}{5.73 \times 10^{-8} \mathrm{~m}} .
$$

This inequality relates the mass transfer resistivity $\hat{r}_{11}$ and the vapor layer thickness $x_{V}$. For resistivities of order unity, the vapor layer would have to be less than $5 \times 10^{-8}$-m wide. Thus, cold to warm mass transfer could not be observed in macroscopic experiments (note that, at such small vapor gaps, the assumptions of continuum theory would not be valid anymore).

However, warm to cold mass transfer is reported for experiments with vapor thicknesses of about $x_{V}=3.5 \mathrm{~mm}$ [22]. To observe warm to cold mass transfer in such a macroscopic cell requires a resistivity value of, at least, $\hat{r}_{11} \simeq 61500$. This required value is 5 orders of magnitude above the value for the resistivity $\hat{r}_{11}$, suggested by kinetic theory. Such an extremely large value for the (dimensionless) resistivity for mass transfer across a phase interface would make itself visible in all macroscopic evaporation experiments: Evaporation would be quite slow, and there would be significant deviation in vapor pressure from the equilibrium saturation pressure for all evaporation experiments. This would be well documented. Indeed, large values of the resistivity $\hat{r}_{11}$ are obtained for a small condensation coefficient $\psi$ in (8). For the 3.5-mm cell, the required value for the condensation coefficient is $\psi<$ $1.5 \times 10^{-5}$. Although the reported values of the condensation coefficients differ by several orders of magnitude, values of the coefficient at such a small order have not been observed elsewhere $[23,24]$.

Cold to warm mass transfer, as reported by Mills and Phillips [2,25,26] and Pursell and Phillips [22] on aniline and water, demands large values of the interface resistivities, in particular, an extremely low condensation coefficient. For the resistivities usually reported for ideal gases, cold to warm heat transfer is impossible. Whereas, some support for resistivities 1 to 2 orders of magnitude larger than values predicted by kinetic theory is reported [9,20], these values would still be too small to allow cold to warm mass transfer. Why the values predicted by kinetic theory $[3-5,17]$ and by molecular dynamics simulations $[18,19]$ are so much smaller than the experimental values, needs further clarification.

\section{INVERTED TEMPERATURE PROFILE}

We finally discuss the meaning of the above solution for the problem of the inverted temperature profile where the total energy flows from warm to cold, but the measurable heat flux $q_{v}$ in the vapor has a direction against the energy flow, cf. Fig. 1 (right panel). In terms of conditions on coefficients $A$ and $B$ in (12) and (13), this is equivalent to

$$
B-A \frac{h_{f g}^{L}}{R T_{L}}=\hat{r}_{11}-\hat{Z} \frac{h_{f g}^{L}}{R T_{L}} \hat{r}_{12}<0 .
$$

Since $\hat{Z} h_{f g}^{L} / R T_{L}$ measures the slope of the saturation pressure curve, the inverted profile is observed as long as the slope is sufficiently steep,

$$
\frac{d \ln p_{\mathrm{sat}}\left(T_{L}\right)}{d \ln T_{L}}=\hat{Z} \frac{h_{f g}^{L}}{R T_{L}}>\frac{\hat{r}_{11}}{\hat{r}_{12}} .
$$

This inequality may be obeyed if the heat of evaporation is large. Far from the critical point where $\hat{Z}=1$, this criterion reduces to the well known condition given in Refs. [3-6]. Here, the criterion has been generalized to states closer to the critical point. Note that this criterion does not depend on the thickness of the vapor layer.

The criterion (25) is sufficient for the observation of the inverted temperature profile. In contrast, the related criterion (20) is a necessary but not a sufficient condition for cold to warm mass transfer. Equation (19) is a sufficient condition and shows that the vapor layer has to be sufficiently thin. We see that the criterion for the inverted $T$ profile invokes the main resistivity to mass transfer $\hat{r}_{11}$ unlike the criterion for cold to warm mass transfer, which invokes $\hat{r}_{22}$, the main interface resistivity to heat transfer. Both criteria invoke the interface coupling coefficient $\hat{r}_{12}$, meaning that both phenomena can be seen as manifestations of the dynamic interface conditions formulated by classical irreversible thermodynamics. 


\section{CONCLUSIONS}

Irreversible thermodynamics provides interface conditions that yield temperature and chemical potential jumps at phase boundaries. The interfacial jumps have, in the past, led to discussions of seemingly unexpected transport phenomena, such as the inverted temperature profile $[3,4]$. To understand this and other phenomena related to the coupled transport of heat and mass at the interface, it is important to solve the transport equations for the whole heterogeneous system, adding the phases adjacent to the interface. Doing this, we have shown here that temperature-difference-driven mass transfer can occur through the vapor phase from a cold to a warm liquid if the heat of evaporation is very low or the resistivity to mass transfer is very high. Observations on cold to warm mass transfer driven by a temperature difference $[22,25,26]$ were conducted for enthalpies of evaporation $h_{f g}^{L}>10 R T_{L}$ and vapor layer thicknesses on the millimeter scale. These conditions may be compatible with the criteria if the resistivity is unphysically high. More data on interfacial resistivities are needed for further progress in the understanding of the dynamics of phase transitions.

\section{ACKNOWLEDGMENTS}

This research was carried out during H.S.'s visit to Trondheim. The visit was made possible through the generous support of the Gas Technology Centre NTNU-SINTEF for which we express our sincere thanks.
[1] S. Kjelstrup and D. Bedeaux, Non-Equilibrium Thermodynamics of Heterogeneous Systems (World Scientific, Singapore, 2008).

[2] C. T. Mills and L. F. Phillips, Chem. Phys. Lett. 372, 615 (2002).

[3] Y. P. Pao, Phys. Fluids 14, 306 (1971).

[4] Y. P. Pao, Phys. Fluids 14, 1340 (1971).

[5] D. Bedeaux, L. J. F. Hermans, and T. Ytrehus, Physica A 169, 263 (1990).

[6] S. Kjelstrup, T. Tsuruta, and D. Bedeaux, J. Colloid Interface Sci. 256, 451 (2002)

[7] G. Fang and C. A. Ward, Phys. Rev. E 59, 417 (1999).

[8] C. A. Ward and G. Fang, Phys. Rev. E 59, 429 (1999).

[9] G. Fang and C. A. Ward, Phys. Rev. E 59, 441 (1999).

[10] V. K. Badam, V. Kumar, F. Durst, and K. Danov, Exp. Thermal Fluid Sci. 32, 276 (2007).

[11] F. Duan, C. A. Ward, V. K. Badam, and F. Durst, Phys. Rev. E 78, 041130 (2008).

[12] M. Bond and H. Struchtrup, Phys. Rev. E 70, 061605 (2004).

[13] J. P. Caputa and H. Struchtrup, Physica A 390, 31 (2011).

[14] S. Kjelstrup, D. Bedeaux, E. Johannessen, and J. Gross, NonEquilibrium Thermodynamics for Engineers (World Scientific, Singapore, 2010).
[15] F. Sharipov, Physica A 203, 437 (1994).

[16] J. Xu, S. Kjelstrup, D. Bedeaux, A. Røsjorde, and L. Rekvig, J. Colloid Interface Sci. 299, 452 (2006).

[17] J. W. Cipolla, H. Lang, and S. K. Loyalka, J. Chem. Phys. 61, 69 (1974).

[18] J. Xu, S. Kjelstrup, D. Bedeaux, A. Røsjorde, and L. Rekvig, J. Colloid Interface Sci. 299, 452 (2006).

[19] J. Ge, S. Kjelstrup, D. Bedeaux, J. M. Simon, and B. Rousseau, Phys. Rev. E 75, 061604 (2007).

[20] D. Bedeaux and S. Kjelstrup, Physica A 270, 413 (1999).

[21] L. V. van der Ham, R. Bock, and S. Kjelstrup, Chem. Eng. Sci. 65, 2236 (2010).

[22] C. J. Pursell and L. F. Phillips, Phys. Chem. Chem. Phys. 8, 4694 (2006).

[23] I. Eames, N. Marr, and H. Sabir, Int. J. Heat Mass Transf. 40, 2963 (1997).

[24] R. Marek and J. Straub, Int. J. Heat Mass Transf. 44, 39 (2001).

[25] C. T. Mills and L. F. Phillips, Chem. Phys. Lett. 366, 279 (2002).

[26] C. T. Mills and L. F. Phillips, Chem. Phys. Lett. 372, 609 (2002). 